\title{
Multiple sulfur isotope analysis of silicate samples by MC-ICP-MS
}

\author{
FELIX GENSKE', SUZETTE TIMMERMAN², SimON \\ SCHURR $^{3}$, HARALD STRAUSS ${ }^{3}$, ANDREAS STRACKE $^{\prime}$ \\ Institut für Mineralogie, WWU Münster, Germany \\ 2Earth and Atmospheric Sciences, University of Alberta, \\ Edmonton, Canada \\ ${ }^{3}$ Institut für Geologie und Paläontologie - Historische und \\ Regionale Geologie, WWU Münster, Germany \\ felix.genske@uni-muenster.de
}

Extreme sulfur isotope heterogeneity in surface reservoirs ${ }^{[1]}$ and differences in isotopic composition between S-species are important for understanding the global S-cycle. During magmatic processes, sulfur affects the oxidation state of a magma, partitioning and transport of metals between different phases, and hydrothermal processes ${ }^{[2]}$. Hence, sulfur isotope variations in magmatic rocks, fluids or gases provide insights into the underlying high-T sulfur cycle.

To date, sulfur isotope measurements in silicate rocks have either been done in-situ on sulfides by secondary ionization mass spectrometry (SIMS) or through combustion of rock powder $(>30 \mu \mathrm{g} \mathrm{S})$ in combination with gas source mass spectrometry. However, there is currently no established method to measure sulfur isotope ratios on small quantities of sulfur from silicate rocks. Here, we present a method for extracting sulfur from rock powders to measure sulfur isotope compositions by multi-collector inductively coupled plasma mass spectrometry (MC-ICP-MS).

Sulfur is leached (80-100\% yield) from powders with aqua regia and purified using anion exchange chromatography (AG1-X8). Isotope measurements are conducted on a Neptune Plus MC-ICP-MS. Use of an ESI APEX- $\Omega$ sample introduction system eliminates oxide and hydride interferences. Isotopically different sulfate solutions reproduce better than $0.15 \%$ for $\delta^{34} S, 0.2 \%$ for $\delta^{33} \mathrm{~S}$, and $0.15 \%$ or $\Delta^{33} \mathrm{~S}$ (2 S.D.) using standard-sample bracketing with a Merck $\mathrm{SO}_{4}$ solution as the bracketing standard $\left(\delta^{34} \mathrm{~S}\right.$ $=+4.4 \% 0 \mathrm{~V}-\mathrm{CDT}$; calibrated by gas source mass spectrometry). Bulk rock $\delta^{34} \mathrm{~S}$ for different silicate reference materials (e.g., BHVO-2, BCR-2, JGb-1, BE-N) reproduce to $0.3-1.3 \%$, indicating sample heterogenity for some materials, and range from $0.9-3.6 \%$ in $\delta^{34} \mathrm{~S}$. Less than 300 ng of $\mathrm{S}$ are required for $\mathrm{S}$-isotope measurements by $\mathrm{MC}$ ICP-MS on silicate samples, and thus facilitate investigation of the high-T sulfur cycle with high spatial resolution.

[1] Canfield and Farquhar (2009) PNAS 106, 8123-8127. [2] Wallace and Edmonds (2011) RMG 73, 215-246. 